\title{
SISTEMA DE CONTROL COOPERATIVO APLICADO A UNA RED DE TRÁFICO URBANO
}

\author{
Antonio Artuñedo, Raúl M. del Toro, Rodolfo Haber \\ Centro de Automática y Robótica (CSIC-UPM), Ctra. M300 Campo Real, Km 0,200, Arganda del Rey \\ \{antonio.artunedo, raul.deltoro, rodolfo.haber\}@car.upm-csic.es
}

\begin{abstract}
Resumen
Hoy en día muchas investigaciones persiguen activamente mejoras en el rendimiento de las redes de tráfico urbano a la vez que la reducción de la concentración de contaminantes en el aire. Un reto fundamental es la necesidad de cooperación entre las diferentes partes de estas redes, tales como vehículos $y$ diferentes sistemas de infraestructura. En este trabajo se aplica un algoritmo para el control cooperativo como solución a problemas relacionados con la movilidad en las ciudades. El objetivo del sistema de control es disminuir la concentración de contaminantes del aire a través de la adaptación de ciclos de semáforos interconectados en red. Para ello el algoritmo emplea información de sistemas de monitorización de la contaminación y del tráfico en la ciudad y se toman decisiones de forma consensuada entre los diferentes dispositivos de control de la red de semáforos. La solución propuesta se ha validado en un entorno simulado y los resultados de las pruebas realizadas corroboran su viabilidad y potencialidad para coordinar las respuestas simultáneas tanto a niveles de contaminación como a flujos de vehículos en redes de tráfico urbano.
\end{abstract}

Palabras Clave: Consenso, Control cooperativo, Polución, Sistemas de eventos discretos, Sistema de Sistemas, Tráfico urbano.

\section{INTRODUCCIÓN}

En la actualidad, el reto crear ciudades sostenibles y respetuosas con el medio ambiente está impulsando las actividades de muchas partes interesadas como las autoridades locales y nacionales, el sector privado, grupos de presión ambientales, institutos de investigación universitarios y asociaciones de vecinos. Están promoviendo conjuntamente soluciones basadas en el enfoque de Sistema de Sistemas (SoS, System-of-systems) para ciudades inteligentes $[6,7,13]$. Desde un punto de vista físico y de ingeniería de sistemas, una ciudad o una región puede ser vista como un sistema físico compuesto por varios subsistemas físicos acoplados (ej., transporte, sistemas de distribución de energía, sistemas de abastecimiento de agua, etc.). El comportamiento dinámico general de la ciudad está determinado a su vez por el comportamiento de estos subsistemas físicos acoplados que son a la vez complejos e inciertos. Además, se imponen requisitos más estrictos para la sostenibilidad, la disponibilidad de recursos, y el funcionamiento óptimo. La capacidad de anticipar y controlar situaciones diarias (por ejemplo, la congestión del tráfico y los altos niveles de contaminación) así como eventos inesperados (por ejemplo, apagones, accidentes de tráfico y las condiciones climáticas excepcionales) será clave para tener éxito en tales condiciones complejas y potencialmente inestables. Mientras tanto, un número creciente de sensores, actuadores, sistemas de comunicación, y ordenadores de bajo coste se encuentran en nuevas aplicaciones que, además de los subsistemas específicos, son capaces de gestionar sistemas urbanos complejos o permitir la cooperación entre ellos.

\subsection{ENFOQUE DE SISTEMA DE SISTEMAS}

La investigación actual en las áreas tecnológicas sobre las ciudades inteligentes, pretende integrar los subsistemas urbanos y hacerlos trabajar de forma conjunta [6]. Estos subsistemas son por lo general de naturaleza diversa, es decir, sistemas con diferentes dominios o diferentes aspectos temporales, tales como los sistemas de información de contaminación y los de control de tráfico. Sin embargo, su comportamiento está de alguna manera relacionado entre sí. La heterogeneidad de los sistemas supone nuevos retos para el enfoque de diseño basado en modelos.

El concepto de Sistema de Sistemas surge de la necesidad de abordar la complejidad de sistemas emergentes interconectados. En este contexto cabe destacar que usualmente los sistemas están diseñados independientemente, evolucionan de forma independiente y pueden funcionar de forma autónoma [1, 3]. Los sistemas que constituyen un SoS tienen sus propios objetivos individuales, mientras que todo el SoS tiene objetivos globales. El objetivo previsto de sub-sistemas que operan 
conjuntamente es un mejor funcionamiento global en comparación con cada sistema considerado individualmente. Los subsistemas también son operativamente independientes y autónomos y las interacciones entre ellos son generalmente asíncronas, por lo que pueden ser representados como modelos de eventos discretos [17].

La solución para el control cooperativo de subsistemas urbanos puede ser diseñada a través de dos esquemas principales: coordinación centralizada y distribuida [16]. En un esquema de coordinación centralizada, se supone que cada sistema tiene la capacidad de comunicarse con un sistema central o compartir información a través de una red completamente conectada. Por el contrario, los esquemas de coordinación distribuidos reducen los requisitos de comunicación utilizando las interacciones locales vecino-a-vecino; mejorando la escalabilidad, la flexibilidad, la fiabilidad y la robustez del funcionamiento conjunto.

\subsection{TOMA DE DECISIONES BASADA EN CONSENSO}

El consenso es un problema fundamental en el estudio de control cooperativo para la coordinación distribuida multi-agente. Nanayakkara et al. [12], proponen el control basado en consenso como un paradigma de control cooperativo para SoS, con el objetivo de extraer mayores beneficios de los sistemas que componen un SoS. Este enfoque contempla un conjunto de subsistemas que persiguen tanto sus propios objetivos particulares como los objetivos comunes, haciendo uso de comunicaciones entre los subsistemas para ello.

Los algoritmos de consenso han sido ampliamente aplicados a los sistemas cooperativos distribuidos, tales como grupos de vehículos [5, 16], enjambres de robots [9], sistemas de control de tráfico [10, 18, 20], o redes de sensores inalámbricos [8], optimización de tráfico y control en entornos urbanos [4, 19], entre otros. En estos casos, el control por consenso permite a cada sistema o agente individual conocer la meta global y, a continuación, en función del estado de los otros componentes del sistema, decidir la mejor acción de control local en la búsqueda de ese objetivo. El problema de optimización del tráfico en entornos urbanos, basada en información de la contaminación, se puede abordar estableciendo un consenso entre los diferentes agentes de control involucrados [4]. En un problema similar (gestión y control del tráfico urbano), Castro et al. propuso un modelo distribuido de múltiples capas para el control predictivo del tráfico urbano. El sistema de control se compone de capas de control local y global donde cada agente se encarga de los semáforos en una intersección. El sistema utiliza los principios de consenso entre los agentes de control y coordinación del comportamiento, con el fin de conciliar los objetivos de control locales y globales.

\subsection{ENFOQUE PROPUESTO}

En este trabajo se aplica un algoritmo de control cooperativo en un escenario urbano, con el fin de proporcionar soluciones a los problemas relacionados con la movilidad y la contaminación en las ciudades. El propósito de este escenario es el de reducir la contaminación del aire en regiones específicas de la ciudad mediante la adaptación de los ciclos de los semáforos en cada intersección. A tal efecto, el servicio de información de la contaminación del aire coopera con el subsistema de control del tráfico. Debido a la naturaleza distribuida de los subsistemas que se contemplan, se aplica un algoritmo de control distribuido basado en consenso. Los niveles de contaminación y densidades de tráfico se consideran entradas de la estrategia de control propuesto, para la cooperación de los subsistemas. Por otra parte, se aplica el formalismo DEVS (Discrete event systems specification) para fines de modelado y simulación. Este marco es adecuado para el modelado de sistemas dinámicos complejos y sus interacciones.

Las principales contribuciones de este trabajo incluyen el diseño y evaluación en un entorno de simulación de una estrategia para disminuir los niveles de contaminación en el tráfico urbano por medio de un enfoque de control cooperativo basado en consenso.

Las siguientes secciones describen el proceso que hemos seguido. La sección 2 presenta el problema que abordamos. La subsección 2.1 hace referencia a la técnica de modelado usada (modelado DEVS). La sección 3 muestra una introducción al control cooperativo basado en consenso y presenta la solución de control cooperativo propuesta. En la sección 4, se describen las simulaciones en lazo abierto $y$ en lazo cerrado. Por último, las conclusiones se presentan en la sección 5 .

\section{ESCENARIO Y DESCRIPCIÓN DE LA SOLUCIÓN}

El escenario propuesto implica diferentes subsistemas urbanos: Control de tráfico y servicios de información de contaminación atmosférica. Se basa en una aplicación de movilidad inteligente en una red interconectada de control de semáforos (TLC, Traffic-light controller) que utiliza información de un subsistema de monitorización del tráfico y un subsistema de monitorización de la contaminación. El objetivo es equilibrar los niveles de contaminación atmosférica en la ciudad mediante la adaptación de 
los ciclos de semáforos. No solo el tráfico influye en los niveles de contaminación de NOx de la ciudad sino que otros subsistemas urbanos también contribuyen al aumento y la caída de los mismos, lo que aumenta la complejidad del problema. La figura 1 muestra un diagrama general del escenario.

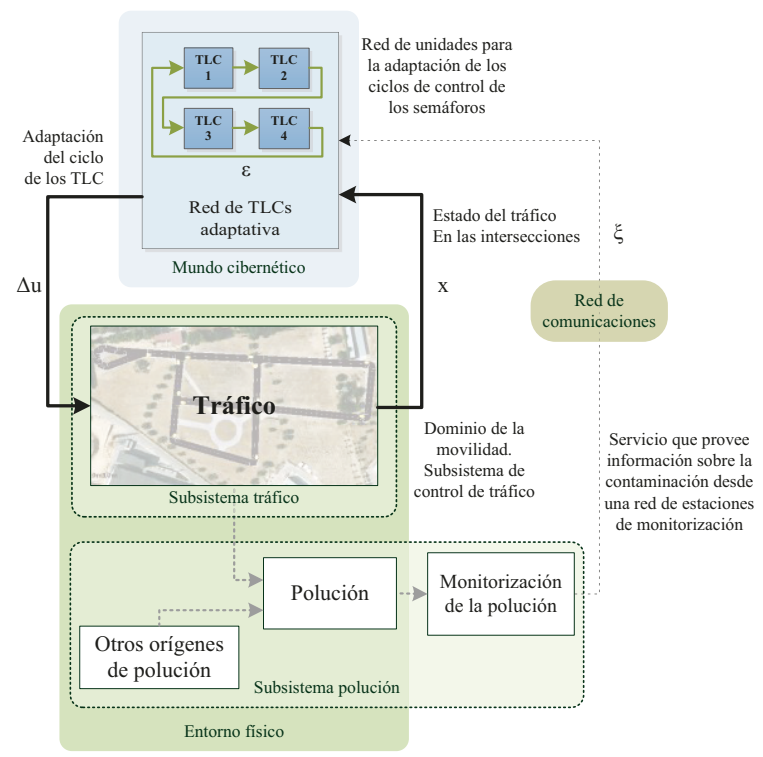

Figura 1: Diagrama general del escenario

La contaminación atmosférica en la ciudad se mide por un sistema de monitorización que proporciona una medición para cada distrito de la ciudad $(\xi)$. Esta medición se envía a las unidades de TLC. En aras de la claridad, el escenario se centra en un distrito, de manera que cada intersección estima su contribución a la contaminación general del aire $(\varepsilon)$ en base a sus colas de vehículos $(X)$. En cuanto a la medición de la información de tráfico, cada intersección tiene sus propios detectores de vehículos para la obtención de información de tráfico. Basándose en información sobre el tráfico y sobre la contaminación del distrito, un algoritmo de control distribuido toma decisiones sobre la conveniencia de actualizar los ciclos de semáforos $(U)$ e informa a las unidades $T L C$ en consecuencia. Las siguientes subsecciones abordan el modelado, diseño de control y simulación de todo el sistema.

\subsection{MODELADO DEVS}

Seleccionamos DEVS como marco para el modelado y simulación de $\mathrm{SoS}$ con el fin de estudiar el comportamiento dinámico y la interacción entre los diferentes componentes del escenario. Este formalismo puede ser utilizado para el modelado y simulación de sistemas dinámicos complejos y sus interacciones [18]. El enfoque basado en el modelado DEVS permite la especificación de modelos básicos y también de la forma en que están conectados entre sí. Estos modelos básicos, también llamados modelos atómicos, son sistemas modulares que reciben entradas (a través de los puertos de entrada), cambian sus estados, y generan salidas (a través de los puertos de salida) en un período de tiempo. Los acoplamientos entre los modelos atómicos dan lugar los llamados modelos acoplados. En este trabajo, aplicamos el formalismo Parallel-DEVS (PDEVS). PDEVS resuelve las colisiones entre las transiciones internas y externas que permiten la activación de todos los componentes inminentes y el envío de su salida a otros elementos. Un modelo básico en formalismo PDEVS se puede representar como:

$$
M=\left\langle X_{M}, Y_{M}, S, \delta_{\text {ext }}, \delta_{\text {int }}, \delta_{\text {con }}, \lambda, t_{a}\right\rangle
$$

donde

$X_{M}=\left\{(p, v) \mid p \in\right.$ IPorts, $\left.v \in X_{p}\right\}$ es el conjunto de puertos de entrada y sus valores,

$Y_{M}=\left\{(p, v) \mid p \in\right.$ OPorts, $\left.v \in Y_{p}\right\}$ es el conjunto de puertos de salida y sus valores,

$S$ es el conjunto de estados

$\delta_{i n t}: S \rightarrow S$ es la función de transición interna

$\delta_{\text {ext }}: Q \times X_{M}^{b} \rightarrow S$ es la función de transición externa

$\delta_{\text {con }}: Q \times X_{M}^{b} \rightarrow S$ es la función de transición de confluencia

$Q=\left\{(s, e) \mid s \in S, e \in\left[0, t_{a}\right]\right\}$ es el conjunto total de estados y $e$ es el tiempo transcurrido desde la transición previa

$\lambda: S \rightarrow Y$ es la función de salida

$t_{a}: S \rightarrow R_{0}^{+} \cup \infty$ es la función de avance de tiempo

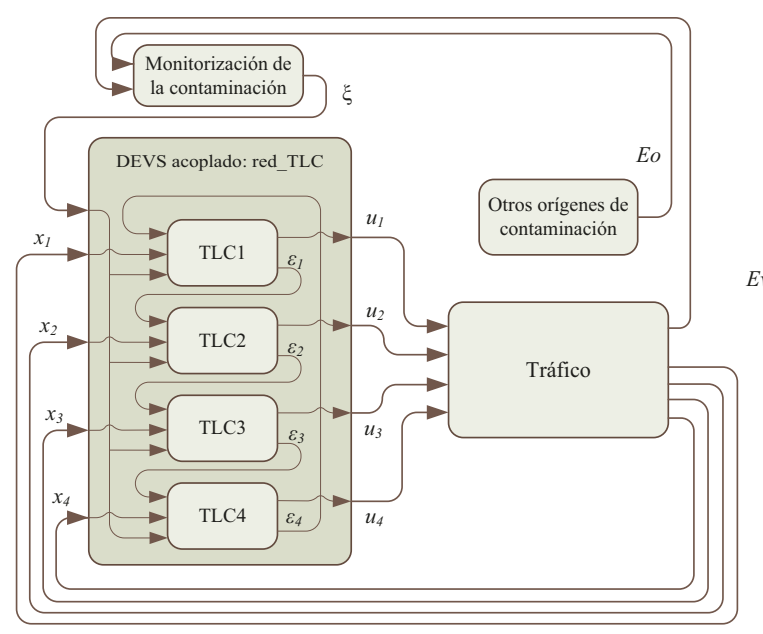

Figura 2: Estructura del modelo DEVS

En esta sección se detalla la representación del sistema basado en el modelo conceptual y los flujos de datos entre los subsistemas. Cada sistema se modeló como un sistema discreto atómico. El acoplamiento de los modelos atómicos forma un 
modelo acoplado. La figura 2 describe los modelos atómicos de cada componente del sistema y sus interconexiones. En el modelo anterior, los siguientes sistemas se definen como modelos atómicos:

- Unidad de control de semáforo (TLC, Trafficlight control unit)

- Servicio de monitorización de contaminación

- Sistema de tráfico (red de carreteras, vehículos, semáforos, etc.)

- Otros orígenes de contaminación

La red interconectada de TLCs comparte información (variable de consenso, $\varepsilon_{i}$ ) para llegar a un consenso entre todos los componentes. Esta red se modela como un modelo acoplado. Cada $T L C$ está conectada con el sistema de Tráfico para adaptar la longitud del ciclo $\left(u_{i}\right)$ de los correspondientes semáforos. La salida del subsistema de tráfico es la información de tráfico (es decir, el flujo de tráfico en cada intersección, $x_{i}$ ) y los datos de contaminación aportados por el tráfico urbano $(E v)$. El sistema llamado "Otros orígenes de contaminación" simula la contribución de la contaminación de otros subsistemas urbanos (por ejemplo, los sistemas de calefacción de edificios, otros sistemas de transporte, etc., Eo). El sistema de monitorización de la contaminación se modela como un sistema de medición de la contaminación urbana que proporciona información de la calidad del aire a la red de $T L C(\xi)$.

Los flujos de datos representados en la figura $1 \mathrm{y}$ figura 2 se describen en la tabla 1.

Tabla 1: Descripción de flujos de datos

\begin{tabular}{|c|l|}
\hline Variable & \multicolumn{1}{c|}{ Descripción } \\
\hline$E v$ & $\begin{array}{l}\text { Monitorización de la emisiones de los } \\
\text { vehículos }\end{array}$ \\
\hline$E o$ & Otras emisiones \\
\hline$\xi$ & $\begin{array}{l}\text { Información de contaminación de la } \\
\text { ciudad. Incluye detalles del estado } \\
\text { actual de la contaminación para una } \\
\text { área geográfica. }\end{array}$ \\
\hline$\varepsilon$ & $\begin{array}{l}\text { Variable de consenso que representa } \\
\text { la dinámica de cada } T L C\end{array}$ \\
\hline$x$ & $\begin{array}{l}\text { Información procesada de los } \\
\text { detectores de tráfico. Es representada } \\
\text { como un vector que hace referencia a } \\
\text { la señal de cada detector. }\end{array}$ \\
\hline$u$ & $\begin{array}{l}\text { Contiene los datos de configuración } \\
\text { de cada } T L C \text {. Incluye los parámetros } \\
\text { requeridos para reconfigurar sus } \\
\text { operaciones. }\end{array}$ \\
\hline
\end{tabular}

\section{DISEÑO DEL CONTROL COOPERATIVO BASADO EN CONSENSO}

La idea principal de un algoritmo de consenso es alcanzar una dinámica similar en los estados de información de cada subsistema [16]. La topología de interacción de una red de agentes se representa usando un grafo dirigido $G=(V, E)$, donde $V=\{1,2, \ldots, n\}$ es un conjunto de nodos finito y no vacío y $E \subseteq V \times V$ es un conjunto de pares de nodos ordenados. $E(i, j)$ denota que el nodo $j$ puede obtener información del nodo $i$. Los vecinos del nodo $i$ son expresados como $N_{i}=\{j \in V:(i, j) \in E\}$. Una forma iterativa del algoritmo de consenso para alcanzar un acuerdo en cuanto al estado de $n$ nodos integradores con dinámica $\dot{x}_{i}=u_{i}$ can puede ser expresado en tiempo discreto como sigue:

$x_{i}(k+1)=x_{i}(k)+\lambda \sum_{j \in N_{i}} a_{i j}\left(x_{j}(k)-x_{i}(k)\right), k \in \mathbb{N}$

Donde $a_{i j}$ es la entrada $(i, j)$ de la matriz de adyacencia $A_{n} \in \mathbb{R}^{n \times n}$ asociada al grafo $G$, y $x_{i}$ es el estado de información del sistema $i$. Una consecuencia de esta ecuación es que el estado de información $x_{i}(k)$ del sistema $i$ es conducido hacia el estado de información de sus vecinos. El consenso es logrado si, para todo $x_{i}(0)$ y para todo $i, j=1, \ldots, n,\left|x_{i}(k)-x_{j}(k)\right| \rightarrow 0$, as $k \rightarrow \infty$.

Los componentes del sistema y las interacciones entre ellos están representados en la figura 3.

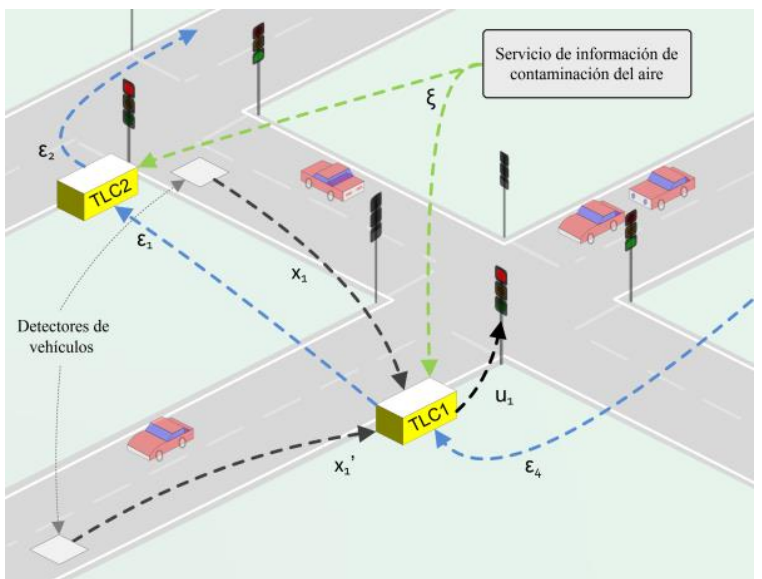

Figura 3: Esquema de interacción entre sistemas

La comunicación entre nodos de red se define a través de un grafo. En este caso, la topología de comunicación se define por un grafo ciclo dirigido compuesto por 4 nodos. 
Se debe definir una variable de consenso que represente la dinámica del sistema para aplicar el control basado en consenso. Cada TLC utiliza información de tráfico local e información de la contaminación proveniente de un servicio de información. Como variable de estado de consenso se tomó una estimación de la concentración de contaminantes en cada intersección. Se ha considerado que la dinámica de cada intersección es la misma y que se comportan como sistemas lineales. El modelo dinámico discreto del nodo, es decir, de la variable de consenso en cada intersección, se define mediante la siguiente ecuación recursiva:

$\varepsilon_{i}(k+1)=\varepsilon_{i}(k)+\alpha_{i} \xi(k-n)+\beta x_{i}(k-m)+\gamma \Delta u_{i}$

donde,

- $\varepsilon_{i}(k)\left[\mathrm{gNO}_{\mathrm{x}} / \mathrm{m}^{3}\right]$ es el estado del sistema $i$ en el instante $k$. El estado del sistema está relacionado con los niveles de contaminación y de tráfico en cada intersección. El contaminante considerado en este estudio es el NOx.

- $\xi(k-n) \quad\left[\mathrm{gNO}_{\mathrm{x}} / \mathrm{m}^{3}\right]$ es una entrada del sistema. Contiene información de contaminación provista a los TLCs en el instante $k-n$ donde $n$ es el retraso entre la emisión de la contaminación y la recepción de la información en cada $T L C$.

- $\alpha_{i}$ es un factor adimensional que representa la contribución de la intersección $i$ a la contaminación general de la ciudad. Su cálculo está basado en la ocupación máxima de la intersección $i$ sobre la ocupación máxima total.

- $x_{i}(k-m)$ [veh] es una entrada del sistema. Contiene la suma de las colas de vehículos en cada aproximación a la intersección controlada por $T L C_{i}$. $m$ representa el retraso entre la medida de la cola de tráfico y la recepción de la información en $T L C_{i}$.

- $\beta\left[\mathrm{gNO}_{\mathrm{x}} / \mathrm{veh} / \mathrm{m}^{3}\right]$ es un factor que caracteriza la emisión de contaminantes de una cola de tráfico dada en una intersección. $\beta=\frac{q \cdot F}{10^{3} \cdot \Delta t}$, donde $q$ es el factor de emisión del contaminante $\left(\mathrm{gNO}_{\mathrm{x}} / \mathrm{veh} / \mathrm{km}\right), F$ es el factor de dispersión del contaminante y $\Delta t$ es el paso de simulación [2]

- $\Delta u_{i} \quad[\%$ T.L. cycle] es la salida del control cooperativo basado en consenso de TLCi. La acción de control es un porcentaje de cambio de la longitud del ciclo del semáforo con respecto al valor inicial, en un rango limitado.

- $\gamma\left[\mathrm{gNO}_{\mathrm{x}} / \mathrm{m}^{3} / \%\right.$ T.L. cycle] representa la influencia de las emisiones de contaminante en los cambios de la temporización de los semáforos. $\gamma=\beta \gamma^{\prime}$, donde $\gamma^{\prime}[\mathrm{veh} / \%$ T.L. cycle] es un factor que caracteriza la longitud de la cola con respecto a cambios en la longitud del ciclo de los semáforo. Este factor asume que la relación es proporcional.

La ley de control adoptada para cada $T L C$ hace uso de información sobre el tráfico y la contaminación así como de información de sus nodos vecinos. Teniendo en cuenta el modelo dinámico considerado en la ecuación 3 y aplicando el control por consenso de la ecuación 2, la expresión de la ley de control queda como sigue:

$\Delta u_{i}(k)=-\frac{1}{\gamma}\left(\alpha_{i} \xi(k-n)+\beta x_{i}(k-m)+\lambda \sum_{j \in N_{i}} a_{i j}\left(\varepsilon_{i}-\varepsilon_{j}\right)\right)$

donde,

- $\quad \lambda$ es un parámetro referido a la estabilidad del sistema. Si $\lambda \in\left(0, \theta^{-1}\right]$, donde $\theta$ es el grado máximo del grafo, la convergencia del algoritmo de consenso está garantizada en un grafo conectado [14].

- $\quad a_{i j}$ es el correspondiente valor de la matriz de adyacencia del grafo.

\section{VALIDACIÓN MEDIANTE SIMULACIÓN}

Para evaluar y validar la solución propuesta se ha empleado un entorno simulado. El escenario de simulación está basado en una red de carreteras que imita un entorno urbano (ver figura 4). Está compuesto por 4 intersecciones semaforizadas ( $\mathrm{J} 1$, $\mathrm{J} 2, \mathrm{~J} 3$ y J4) a través de las que los vehículos circulan siguiendo rutas predefinidas de forma aleatoria en cada simulación. Todos los vehículos empleados han sido definidos del mismo tipo y su comportamiento se basa en un modelo de seguimiento de vehículos con los siguientes parámetros:

- Longitud del vehículo: $5.00 \mathrm{~m}$

- Separación mínima: $2.50 \mathrm{~m}$

- Máxima velocidad: $55.56 \mathrm{~m} / \mathrm{s}$

- Máxima aceleración: $2.60 \mathrm{~m} / \mathrm{s} 2$

- Máxima deceleración: $4.50 \mathrm{~m} / \mathrm{s} 2$

- Imperfección: 0.50

- Tiempo de reacción: $1 \mathrm{~s}$

Para la simulación de tráfico se empleó el simulador microscópico SUMO [11].

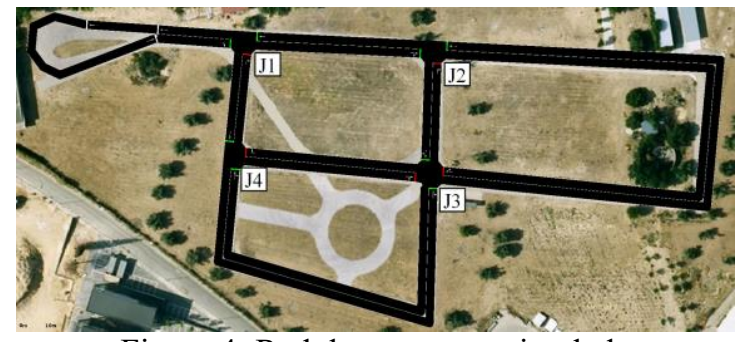

Figura 4: Red de carreteras simulada 


\subsection{SIMULACIÓN EN LAZO ABIERTO}

El escenario de simulación utilizado es el definido en la sección anterior. En este caso, simulación en lazo abierto, las TLCs trabajan con una temporización fija, es decir, no hay control actuando sobre ellas. El tiempo de simulación es de 500 segundos, siendo suficiente para observar la evolución de las distintas variables de interés.

En la figura 5 se distinguen los niveles de tráfico en cada intersección (a) así como la contaminación (b) a lo largo de la simulación. Como se observa, los niveles de contaminación se ven incrementados con el aumento del número de vehículos en cada intersección con el paso del tiempo. Ambas gráficas de la figura 5 comienzan en el segundo 100 ya que al comienzo de la simulación el escenario se encuentra vacío.

\subsection{SIMULACIÓN COOPERATIVO}

DEL

CONTROL

Para la simulación del control cooperativo se empleó el mismo escenario descrito en la sección 4. Para el modelado y la simulación DEVS se utilizó un toolbox para Matlab llamado MatlabDEVS [15]. También se empleó la interfaz TraCI del simulador de tráfico SUMO para la interacción con los modelos DEVS.

En este caso se definieron siguientes parámetros para la simulación del control cooperativo:

1. El contaminante considerado en este trabajo fue el NOx. La cantidad de NOx producida por los vehículos fue tomada del HBEFA ${ }^{1}$, considerando que son vehículos de pasajeros entre 2005 y 2015 (0.35 gNOx/veh/km).

2. El modelo empleado para las emisiones con origen diferente al tráfico consiste en una generación de valores aleatorios a través de una distribución normal con media: $\mu_{\mathrm{Eo}}=30.36$ $\mu \mathrm{gNO}_{\mathrm{x}} / \mathrm{m}^{3}$ y desviación típica $\sigma_{\mathrm{Eo}}=10.48$ $\mu \mathrm{gNO}_{\mathrm{x}} / \mathrm{m}^{3}$. Dichos valores fueron ajustados de forma heurística teniendo en cuenta datos reales de contaminación urbana y contaminación de tráfico. Para el resto de modelos se usaron valores típicos de temporización empleados en sistemas reales. Produce información de contaminación cada 5 segundos $(n=5$, ya que el paso de simulación es de 1 segundo)

\footnotetext{
${ }^{1}$ Handbook Emission Factors for Road Transport (HBEFA, http://www.hbefa.net)
}
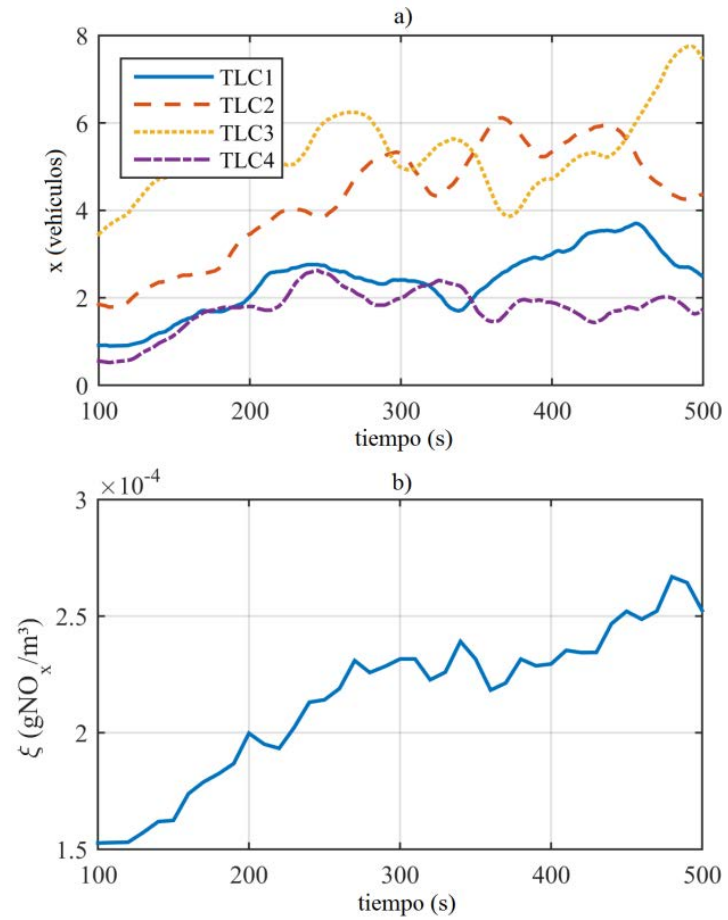

Figura 5: a) Colas de vehículos, b) Evolución de la contaminación en la simulación de lazo abierto

3. El modelo DEVS "Monitorización de la contaminación" filtra los datos de entrada utilizando un filtro de ventana móvil con un tamaño de ventana de 100 segundos y genera información cada 10 segundos $(m=10)$.

4. El periodo de ejecución del modelo DEVS de cada $T L C$ es de 1 segundo. La entrada información de tráfico es filtrada usando un filtro de ventana móvil con un tamaño de ventana de 100 segundos.

5. Dado que el máximo grado del grafo $(\theta)$ es 2 (número máximo de conexiones de un nodo), el valor del factor $\lambda$ para las pruebas de validación fue ajustado a 0.15 para asegurar la estabilidad del control por consenso $(\lambda \in(0,1 / \theta])$.

7. El valor de $\gamma^{\prime}$ fue estimado aplicando regresión lineal con valores obtenidos por simulación. El valor adoptado fue 12.68 [veh/\% T.L. cycle].

Debido a la alta dependencia de las condiciones de tráfico, el escenario fue simulado 420 veces, en condiciones diferentes de tráfico, tanto en lazo abierto como en lazo cerrado. En la figura 6 se muestran los resultados de una de las simulaciones en lazo cerrado. Como se puede observar, la variable de consenso tiende al mismo valor en todas las TLCs con el paso del tiempo. Por otra parte, se observa cómo se estabiliza el nivel de contaminación total (d) 

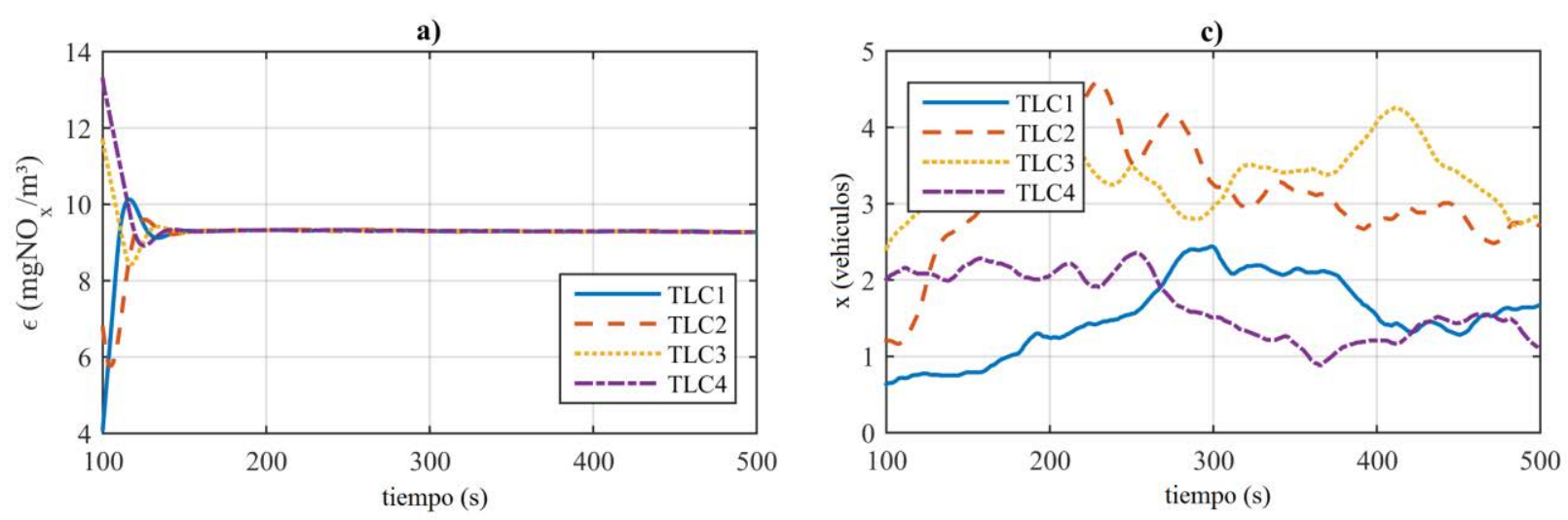

b)
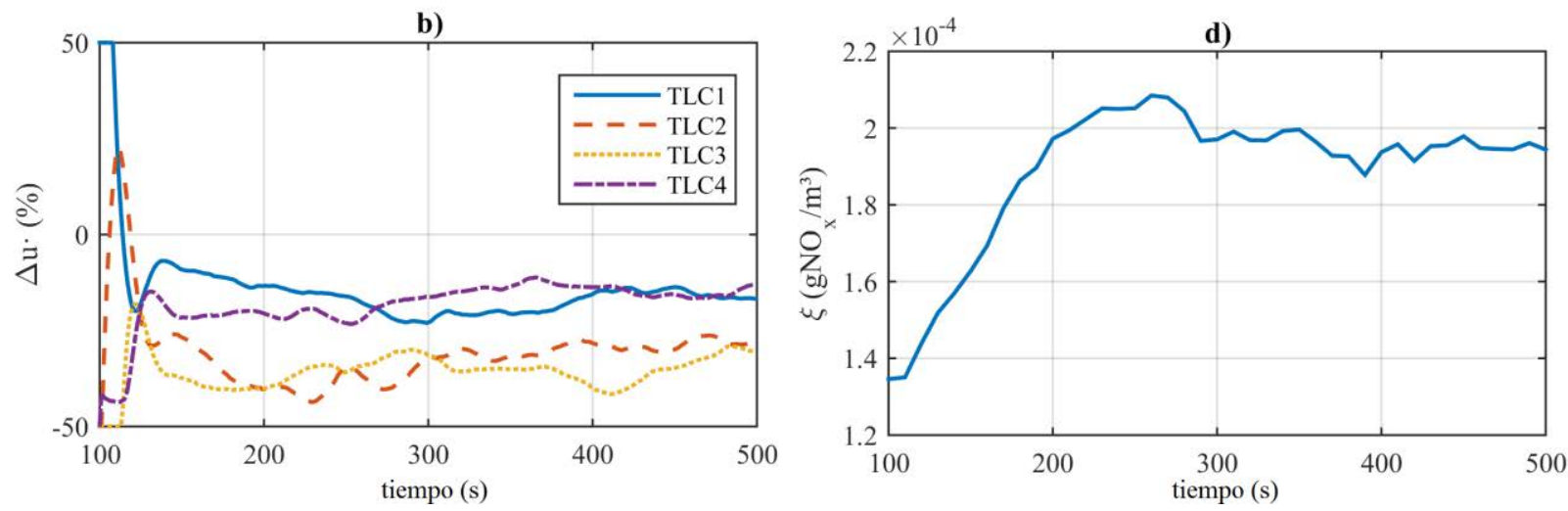

Figura 6: a) Variable de consenso, b) Entrada de control, c) Colas de vehículos, d) Evolución de la contaminación en la simulación de lazo cerrado

como consecuencia de la actuación del sistema de control. Para evaluar el comportamiento del sistema se empleó la integral del valor cuadrático de la contaminación total $\left(\int_{t_{s}}^{t_{f}} \xi^{2} d t\right.$ ), en el intervalo de tiempo de la simulación del escenario $(0-500 \mathrm{~s})$. El valor medio de este índice de todas las simulaciones realizadas fue de 9.52 para lazo abierto y de 0.39 para lazo cerrado, correspondiéndose a una mejora del comportamiento del sistema en lazo cerrado del $95,89 \%$.

\section{CONCLUSIONES}

En este estudio se ha presentado un enfoque para el control cooperativo en un entorno urbano. En esta aplicación, un sistema de control de tráfico usa información de un sistema de monitorización de contaminación para adaptar la longitud del ciclo de semáforos en intersecciones. Un algoritmo de control basado en consenso se ha utilizado para compensar la actuación de cada intersección utilizando información de los agentes vecinos.

Para modelar el sistema en su conjunto se usó el formalismo DEVS. Este paradigma de modelado permite modelar los sistemas individuales a la vez que su interacción con otros sistemas con diferente naturaleza o comportamiento temporal. Además se ha evaluado la solución propuesta en un entorno de simulación y se ha comparado el comportamiento del sistema en lazo abierto y lazo cerrado.

Los resultados de las simulaciones realizadas mostraron la eficacia del sistema de control distribuido propuesto haciendo tender a los diferentes agentes hacia un mismo valor de la variable de consenso y consecuentemente disminuir la concentración de contaminantes, demostrando la viabilidad de la solución propuesta. Teniendo en cuenta estos resultados, se plantea como trabajos futuros continuar con el desarrollo del sistema aprovechando la posible flexibilidad y escalabilidad del sistema.

\section{Agradecimientos}

Este trabajo se ha desarrollado con el apoyo de los proyectos RTC-2015-3942-4 "Family of HighPerformance Compact Cards for Automotive Applications" (TCAP-Auto) y DPI2014-53525-C3-3$\mathrm{R}$ : "Extension of Cognitive Capabilities to the navigation of robots in non-structured Environments" (NAVEGASE), ambos financiados por el Ministerio 
de Econoimía y Competitividad (MINECO) y financiación FEDER.

\section{Referencias}

[1] Adler, J. L. y Blue, V. J., "Toward the design of intelligent traveler information systems", (1998) Transportation Research Part C: Emerging Technologies, vol. 6, pp. 157-172.

[2] Belalcazar, L. C., Fuhrer, O., Ho, M. D., Zarate, E., y Clappier, A., "Estimation of road traffic emission factors from a long term tracer study", (2009) Atmospheric Environment, vol. 43, pp. 5830-5837.

[3] Boardman, J. y Sauser, B., "System of Systems - the meaning of of," in System of Systems Engineering, 2006 IEEE/SMC International Conference on, 2006, p. 6 pp.

[4] Castro, G., Martini, J., y Hirakawa, A., "Multilayer distributed model predictive control of urban traffic", (2013) WIT Transactions on Ecology and The Environment, 179: 967, vol. 976,

[5] Fax, J. A. y Murray, R. M., "Information flow and cooperative control of vehicle formations", (2004) Automatic Control, IEEE Transactions on, vol. 49, pp. 1465-1476.

[6] Gurgen, L., Gunalp, O., Benazzouz, Y., y Gallissot, M., "Self-aware cyber-physical systems and applications in smart buildings and cities," in Design, Automation \& Test in Europe Conference \& Exhibition (DATE), 2013, 2013, pp. 1149-1154.

[7] Harrison, C. y Donnelly, I. A., "A theory of smart cities," in Proceedings of the 55th Annual Meeting of the ISSS-2011, Hull, UK, 2011.

[8] Jeličić, V., Tolic, D., y Bilas, V., "Consensusbased decentralized resource sharing between co-located Wireless Sensor Networks," in Intelligent Sensors, Sensor Networks and Information Processing (ISSNIP), 2014 IEEE Ninth International Conference on, 2014, pp. 16.

[9] Joordens, M. A. y Jamshidi, M., "Consensus Control for a System of Underwater Swarm Robots", (2010) Systems Journal, IEEE, vol. 4, pp. 65-73.

[10] Kim, B. Y. y Ahn, H. S., "Distributed Coordination and Control for a Freeway Traffic Network Using Consensus Algorithms", (2016) IEEE Systems Journal, vol. 10, pp. 162-168.

[11] Krajzewicz, D., Erdmann, J., Behrisch, M., y Bieker, L., "Recent development and applications of SUMO-simulation of urban mobility", (2012) International Journal On Advances in Systems and Measurements, vol. 5,

[12] Nanayakkara, T., Sahin, F., y Jamshidi, M., Intelligent control systems with an introduction to system of systems engineering: CRC Press, 2010.

[13] Naphade, M., Banavar, G., Harrison, C., Paraszczak, J., y Morris, R., "Smarter Cities and Their Innovation Challenges", (2011) Computer, vol. 44, pp. 32-39.

[14] Olfati-Saber, R., Fax, J. A., y Murray, R. M., "Consensus and Cooperation in Networked Multi-Agent Systems", (2007) Proceedings of the IEEE, vol. 95, pp. 215-233.

[15] Pawletta, T., Deatcu, C., Pawletta, S., Hagendorf, O., y Colquhoun, G., "DEVS-based modeling and simulation in scientific and technical computing environments", (2006) SIMULATION SERIES, vol. 38, p. 151.

[16] Ren, W. y Beard, R., Distributed consensus in multi-vehicle cooperative control: theory and applications: Springer, 2007.

[17] Rouphail, N., Tarko, A., y Li, J., "Traffic flow at signalized intersections",

[18] Wang, Z. y Sui, C., "Distributed traffic network control system," in Signal Processing, Communication and Computing (ICSPCC), 2013 IEEE International Conference on, 2013, pp. 1-4.

[19] Wuthishuwong, C. y Traechtler, A., "Consensus Coordination in the Network of Autonomous Intersection Management," 2014.

[20] Zhonghe, H., Yangzhou, C., Jianjun, S., Xu, W., y Jizhen, G., "Consensus based Approach to the Signal Control of Urban Traffic Networks", (2013) Procedia - Social and Behavioral Sciences, vol. 96, pp. 2511-2522. 